\title{
Zur 41.Jahrestagung der Deutschsprachigen Arbeitsgemeinschaft für Mikrochirurgie der peripheren Nerven und Gefäße (DAM) vom 21. bis 23. November 2019 an der Ludwig-Maximilians - Universität München
}

Die 41. Jahrestagung der Deutschsprachigen Arbeitsgemeinschaft für Mikrochirurgie der peripheren Nerven und Gefäße (DAM) wurde vom 21. bis 23. November 2019 von der Abteilung für Handchirurgie, Plastische Chirurgie und Ästhetische Chirurgie der Ludwig-Maximilians Universität München am historischen Campus Innenstadt ausgerichtet [1]. Mit 211 Teilnehmern aus 6 Ländern und insgesamt 20 Ausstellern und Sponsoren konnte die internationale Tagung erfolgreich abgeschlossen werden ( $\triangleright$ A bb. 1). Die jährlich steigende Anzahl an Kongressteilnehmern ist Beleg für die weiterhin zunehmende Bedeutung und hohe Aktualität der Mikrochirurgie und bestätigt die positive Entwicklung des Fachgebietes Plastische Chirurgie in den letzten Jahrzehnten.

Seit jeher zeichnen sich die Jahrestagungen der DAM durch ein ausgewähltes wissenschaftliches Programm und intensive fachliche Interaktion eben im Sinne einer Arbeitsgemeinschaft aus. Dieser Charakter wurde auch bei der diesjährigen Tagung beibehalten. Für die Tagung in München wurden durch das wissenschaftliche Komitee insgesamt 78 Vorträge zu wichtigen Forschungsthemen wie Plexus brachialis und Nervenchirurgie, Brustrekonstruktion und Mikrochirurgie bei Lymphödem akzeptiert. Die anschließenden, konstruktiven Diskussionen waren Beleg für die Aktualität der Themen. Zur Verbesserung des E-Learnings wurden die Vorträge mitgeschnitten und im Mitgliederbereich der DAM zur Verfügung gestellt. Als wesentliche Neuerung wurden an zwei Tagen insgesamt acht Instruktionskurse, die speziell auf den Wissenstransfer an die jüngeren Mikrochirurgen zu generellen Themenkomplexen abzielten, angeboten. In diesen, als „Early Morning Lecture“ zusätzlich zum Hauptprogramm konzipierten, Sitzungen wurden in Gruppen von 10 bis 25 Teilnehmern konkrete Fragen zu verschiedenen Themengebieten der Mikrochirurgie, wie z. B. Rekonstruktive Brustchirurgie, Sarkomchirurgie oder Nervenrekonstruktion, intensiv diskutiert, was einen sehr effekti-

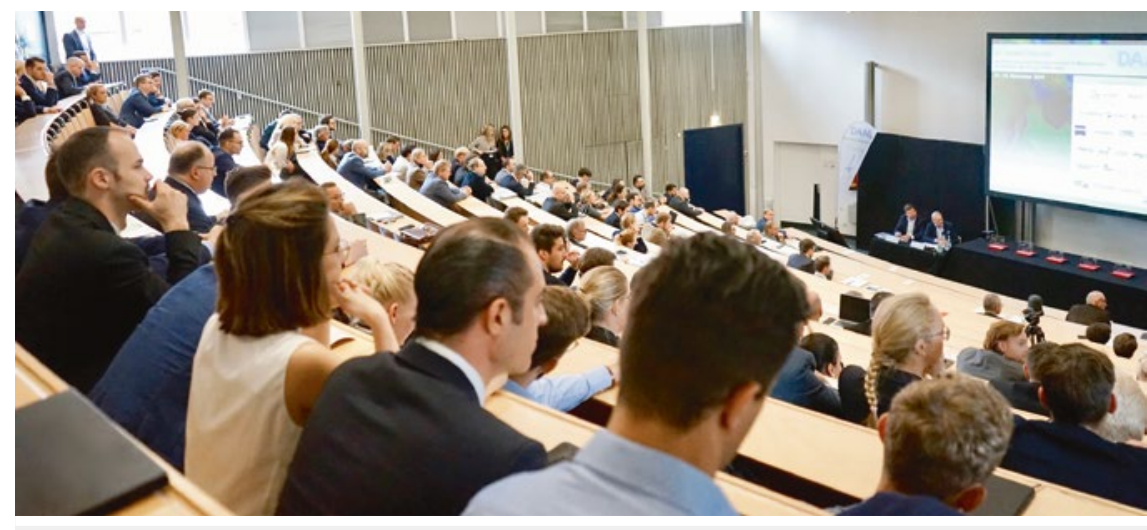

- Abb.1211 Teilnehmer aus Deutschland, Österreich, der Schweiz, den USA und verschiedenen anderen Ländern bei der internationalen 41. Jahrestagung der DAM in München.

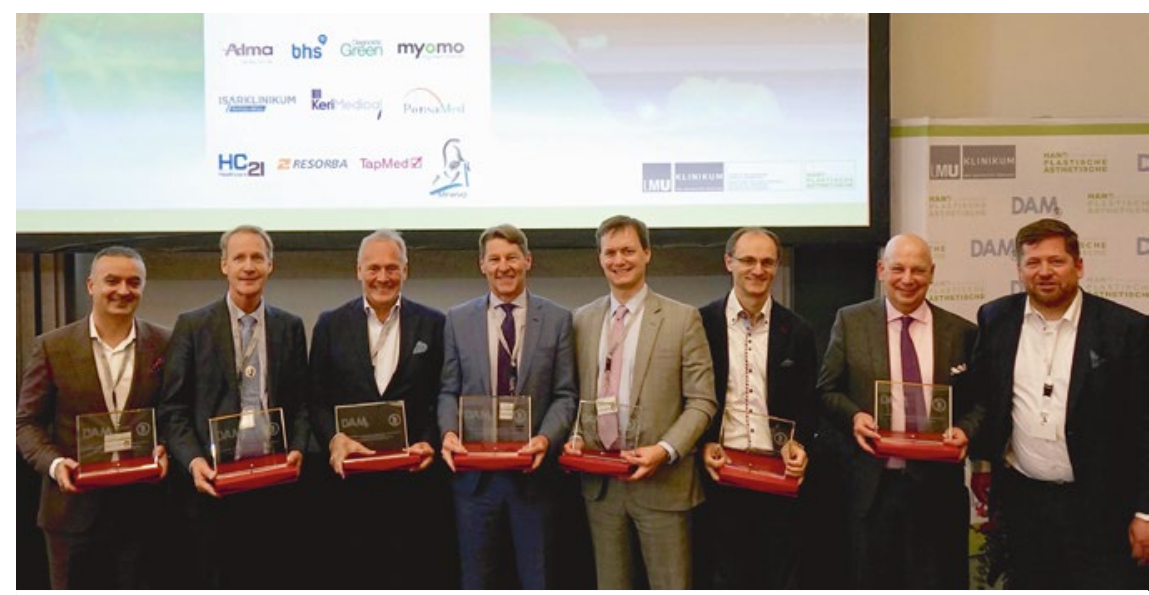

Abb.2 Die Gastredner der American Society of Reconstructive Surgery (ASRM) in der „Best of ASRM“ Sitzung. V. I. n. r.: Prof. Dr. Bauback Safa, Prof. Dr. Michael Neumeister, Prof. Dr. Günter Germann, Prof. Dr. Paul Cederna, Prof. Dr. Patrick Garvey, Prof. Dr. Ivica Ducic, Prof. Dr. Scott Levin und Prof. Dr. Riccardo Giunta (Tagungspräsident).

ven Wissensaustausch ermöglichte. Insbesondere aufgrund der ausgewählten nationalen wie internationalen Kursleiter wurde das neue Format trotz der frühen Uhrzeit mit über 100 Teilnehmern sehr gut angenommen.

Das Herzstück der Jahrestagungen sind bereits seit langer Zeit die Workshops, in denen jährlich Konsensuspapiere erarbeitet werden. Die Beschlüsse leisten einen wichtigen Beitrag zur weiteren Standardisierung der mikrochirurgischen Routine im deutschsprachigen Raum und werden traditionsgemäß in der vorliegenden wissenschaftlichen Zeitschrift veröffentlicht [2-4]. Die Themen der diesjährigen Workshops lauteten:

- „Präoperative Diagnostik bei Perforatorlappen - Duplexsonografie, CT/MR Angiografie oder gar nichts?" (Vorsitz: Prof. Dr. Ulrich Kneser)

- „Perioperatives Management in der Mikrochirurgie“ (Vorsitz: Prof. Dr. Marcus Lehnhardt)

- „Wirtschaftliche Aspekte in der Mikrochirurgie“ (Vorsitz: Prof. Dr. Steffen Eisenhardt) 


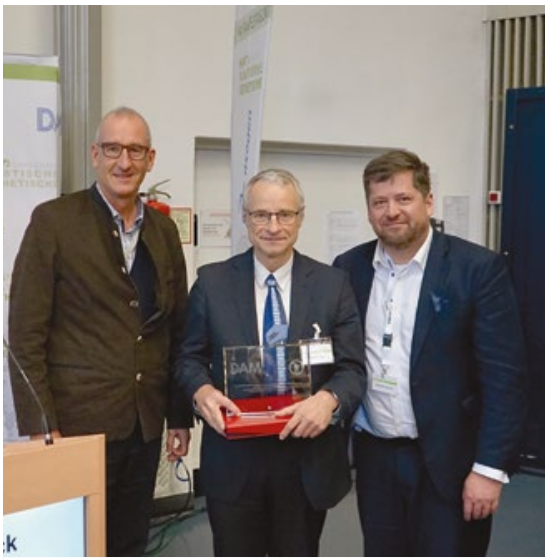

-Abb. 3 Ehrenvorlesung „Mein persönlicher Rückblick auf ein Berufsleben als Rekonstruktiver Mikrochirurg“. V. I. n. r.: Prof. Dr. Dirk Schaefer (Präsident der DAM), Prof. Dr. Bernd Rieck und Prof. Dr. Riccardo Giunta (Tagungspräsident).

Durch die Vorsitzenden und Vortragenden wurde der aktuelle Wissensstand vorgestellt, während der folgenden Diskussion konnten wichtige Beschlüsse zu diesen aktuellen Themen erarbeitet werden. Die Ergebnisse der Workshops erhoffen wir uns noch dieses Jahr im Rahmen von Konsensuspapieren in der HaMiPla im Open Access lesen zu können. Darüber hinaus sind sie über die Webseite der DAM im Download verfügbar (https://dam-mikrochirurgie. org).

Erstmals wurde weiterhin eine Gastgesellschaft zum weiteren internationalen Wissenstransfer eingeladen. Mit der American Society of Reconstructive Microsurgery (ASRM) konnte eine der renommiertesten nationalen Gesellschaften für Mikrochirurgie gewonnen werden. Insbesondere Herrn Prof. Dr. Günter Germann sei an dieser Stelle gedankt. Als „Incoming President“ der ASRM hat er wesentlich zur Organisation und zum Gelingen dieser Sitzung beitragen können. Die „Best of ASRM“ Sitzung war ein Höhepunkt der Tagung, für die sechs führende Mitglieder der ASRM als Vortragende gewonnen werden konnten ( $\triangleright \mathbf{A b b} . \mathbf{2})$ :

- Prof. Dr. Paul Cederna (University of Michigan, Ann Arbor)

- Prof. Dr. Ivica Ducic (The George Washington University, McLean/ Washington D.C.)

- Prof. Dr. Patrick Garvey (The University of Texas MD Anderson Cancer Center, Houston)

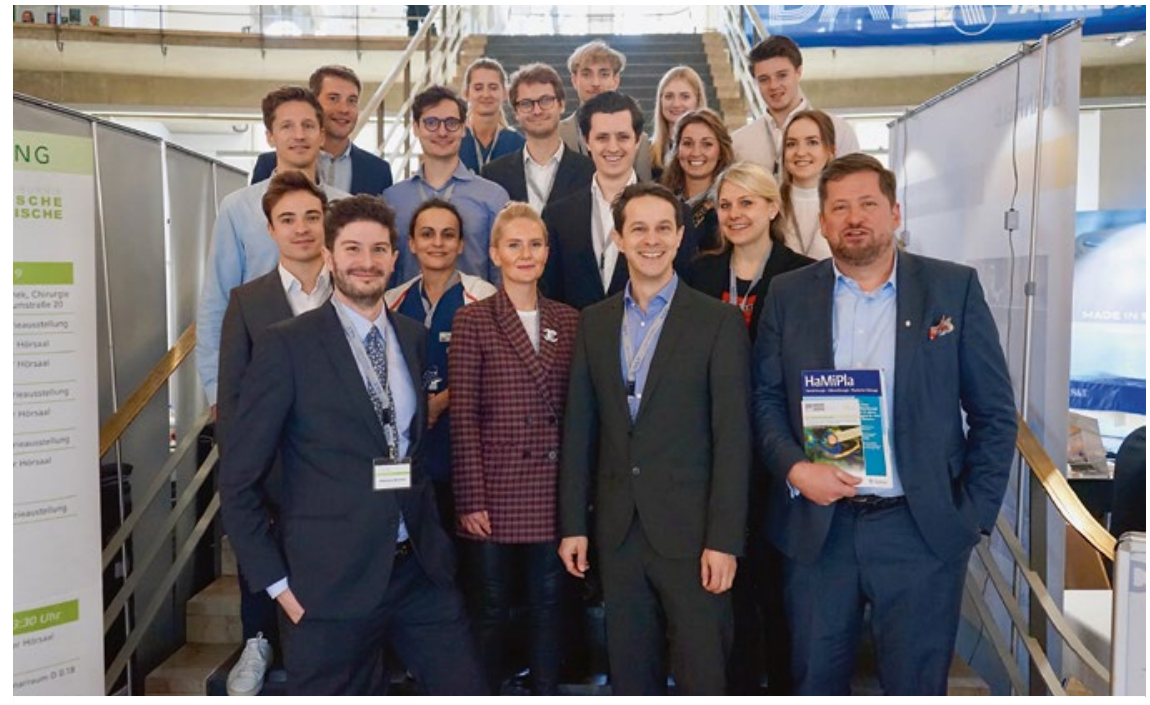

-Abb.4 Organisationsteam der 41. Jahrestagung der DAM in München an der LudwigMaximilians Universität München

- Prof. Dr. Scott Levin (University of Pennsylvania, Philadelphia)

- Prof. Dr. Michael Neumeister (Southern Illinois University, Springfield)

- Prof. Dr. Bauback Safa (The Buncke Clinic, San Francisco)

In den englischsprachigen Vorträgen wurden neueste Forschungsergebnisse und operative Techniken der US-amerikanischen Kollegen vorgestellt, unter anderem zu Allotransplantation von Extremitäten [5, 6], Gewebekonditionierung von Lappenplastiken [7, 8] und zur virtuellen Planung mikrochirurgischer Rekonstruktionen im Kopf-Hals-Bereich [9, 10]. Den Kollegen sei an dieser Stelle für Ihre hervorragenden Beiträge gedankt.

Die diesjährige Ehrenvorlesung wurde von Prof. Dr. Bernd Rieck gehalten. In seinem Vortrag gab Prof. Dr. Rieck einen Rückblick auf ein erfolgreiches Berufsleben als rekonstruktiver Mikrochirurg und ermöglichte so insbesondere jüngeren Kollegen einen persönlichen und umfassenden Einblick in das Fachgebiet ( $>$ Abb. 3). In der Sitzung, „DAM Best Case Battle“, stellten insgesamt zehn ausgewählte Referenten komplexe klinische Fälle vor. Der Vortrag „Die Rolle der Sparepart-Chirurgie bei primären mikrochirurgischen Rekonstruktionen nach Verbrennungsverletzungen “ von Omid Amiri aus Leipzig wurde als bester Beitrag dieser Sitzung ausgezeichnet. Als bester wissenschaftlicher Vortrag der Tagung wurde der Beitrag von Dr. Florian Früh aus Zürich nominiert. Dr. Früh stellte mit seinem Vortrag „Mikrovaskuläre Fragmente aus Fettgewebe induzieren Lymphangiogenese in einem Lymphödemmodell am Hinterlauf der Maus" neueste Forschungsergebnisse zur Behandlung des Lymphödems vor [11].

Während den Sitzungen, Workshops und Instruktionskursen sowie an den fünf bereitgestellten mikrochirurgischen Arbeitsplätzen des „Frau Professor Schmidt-Tintemann Labors für Mikrochirurgie der LMU München“ konnte ein überaus lebendiger wissenschaftlicher Austausch beobachtet werden. Gerade auch die Anzahl an teilnehmenden Studenten bestätigt die Faszination Mikrochirurgie für die Nachwuchsgenerationen.

Die hohe Teilnehmerzahl, die internationalen Referenten und Teilnehmer sowie das hochwertige wissenschaftliche Programm der 41. Jahrestagung bestätigen die positive Entwicklung in der Mikrochirurgie im deutschsprachigen Raum und schaffen beste Voraussetzungen für eine erfolgreiche Tagung im November 2020 in Klagenfurt am Wörthersee. Dem gesamten Team der Abteilung für Handchirurgie, Plastische Chirurgie und Ästhetische Chirurgie der Ludwig-Maximilians Universität sei an dieser Stelle gedankt ( $\mathbf{A} \mathbf{A b} \mathbf{b} \mathbf{4}$ ). 
Autorinnen/Autoren

Nikolaus C. Wachtel

Thilo L. Schenck

Riccardo E. Giunta

\section{Korrespondenzadresse}

Riccardo E. Giunta

Abteilung für Handchirurgie, Plastische

Chirurgie und Ästhetische Chirurgie

Klinikum der Ludwig-Maximilians

Universität München

Pettenkoferstr. 8a

80336 München

Tel.: + 49-89-4400-52697

E-Mail: r.giunta@med.uni-muenchen.de

\section{Literatur}

[1] Giunta RE. Mikrochirurgie im Fokus. Handchir Mikrochir Plast Chir 2019; 51 (6): 409. doi: 10.1055/a-0976-2165. Epub 7.11.2019

[2] Eisenhardt SU, Momeni A, von Fritschen U et al. Brustrekonstruktion mit freien TRAM oder DIEP Lappen - Was ist zeitgemäßer Standard? Handchirurgie, Mikrochirurgie, plastische Chirurgie: Organ der Deutschsprachigen Arbeitsgemeinschaft für Handchirurgie: Organ der Deutschsprachigen Arbeitsgemeinschaft für Mikrochirurgie der Peripheren Nerven und Gefässe 2018; 50 : 248-255. doi:10.1055/a-0631-9025

[3] Hirche C, Engel H, Seidenstuecker K et al. Rekonstruktive Mikrochirurgie des sekundären Lymphödems: Konsensus der Deutschsprachigen Arbeitsgemeinschaft für Mikrochirurgie der peripheren Nerven und Gefäße (DAM) zur Indikation, Diagnostik und Therapie mittels Lymphovenöser Anastomosen (LVA) und vaskularisierter Lymphknotentransplantation (VLKT). Handchirurgie, Mikrochirurgie, plastische Chirurgie: Organ der Deutschsprachigen Arbeitsgemeinschaft für Handchirurgie: Organ der Deutschsprachigen Arbeitsgemeinschaft für Mikrochirurgie der Peripheren Nerven und Gefässe 2019; 51: 424-433. doi:10.1055/a-0874-2212

[4] Schmauss D, Beier JP, Eisenhardt SU et al. Der sichere Lappen - Präoperatives GefäßMapping und intraoperative Perfusionsmessung zur Reduktion der lappenbedingten Morbidität. Handchirurgie, Mikrochirurgie, plastische Chirurgie: Organ der Deutschsprachigen Arbeitsgemeinschaft für Handchirurgie: Organ der Deutschsprachigen Arbeitsgemeinschaft für Mikrochirurgie der Peripheren Nerven und Gefasse 2019; 51: 410-417. doi:10.1055/a-0987-0118

[5] Levin LS. From Autotransplantation to Allotransplantation: A Perspective on the Fu- ture of Reconstructive Microsurgery. Journal of reconstructive microsurgery 2018; 34: 681-682. doi:10.1055/s-0038-1639367

[6] Mendenhall SD, Brown S, Ben-Amotz $O$ et al. Building a Hand and Upper Extremity Transplantation Program: Lessons Learned From the First 20 Years of Vascularized Composite Allotransplantation. Hand (New York, NY) 2018. doi:10.1177/1558944718790579: 1558944718790579 .

[7] Daugherty THF, Pribaz JJ, Neumeister MW. The Use of Prefabricated Flaps in Burn Reconstruction. Clinics in plastic surgery 2017; 44: 813-821. doi:10.1016/j. cps.2017.05.012

[8] Houle JM, Neumeister MW. A prefabricated, tissue-engineered Integra free flap. Plastic and reconstructive surgery 2007; 120: 1322-1325. doi:10.1097/01. prs.0000279495.73662.ca

[9] Chang El, Boukovalas S, Liu J et al. Reconstruction of Posterior Mandibulectomy Defects in the Modern Era of Virtual Planning and Three-Dimensional Modeling. Plastic and reconstructive surgery 2019; 144: 453e-462e. doi:10.1097| prs.0000000000005954

[10] Largo RD, Garvey PB. Updates in Head and Neck Reconstruction. Plastic and reconstructive surgery 2018; 141: 271e-285e. doi: $10.1097 /$ prs. 0000000000004070

[11] Frueh FS, Spater T, Scheuer $C$ et al. Isolation of Murine Adipose Tissue-derived Microvascular Fragments as Vascularization Units for Tissue Engineering. Journal of visualized experiments: JoVE 2017. doi:10.3791/55721. doi: $10.3791 / 55721$ 